\title{
GALOIS H-OBJECTS WITH A NORMAL BASIS IN CLOSED CATEGORIES. A COHOMOLOGICAL INTERPRETATION
}

\author{
J. N. Alonso Álvarez and J. M. Fernández Vilaboa
}

Abstract

In this paper, for a cocommutative Hopf algebra $\mathbf{H}$ in a symmetric closed category $\mathcal{C}$ with basic object $K$, we get an isomorphism between the group of isomorphism classes of Galois H-objects with a normal basis and the second cohomology group $H^{2}(\mathrm{H}, K)$ of $\mathrm{H}$ with coefficients in $K$. Using this result, we obtain a direct sum decomposition for the Brauer group of $\mathrm{H}$-module Azumaya monoids with inner action:

$$
\mathrm{BM}_{\mathrm{inn}}(C, \mathrm{H}) \cong \mathrm{B}(C) \oplus H^{2}(\mathrm{H}, K)
$$

In particular, if $\mathcal{C}$ is the symmetric closed category of $K$-modules with $K$ a field, $H^{2}(H, K)$ is the second cohomology group introduced by Sweedler in [21]. Moreover, if $H$ is a finitely generated projective, commutative and cocommutative Hopf algebra. over a commutative ring with unit $K$, then the above decomposition theorem is the one obtaned by Beattie [ 5 ] for the Brauer group of $H$-module algebras.

\section{Preliminary}

A monoidal category $(\mathcal{C}, \otimes, K)$ consists of a category $\mathcal{C}$ with a bifunctor $-\otimes-: \mathcal{C} \times \mathcal{C} \rightarrow \mathcal{C}$ and a basic object $K$, and with natural isomorphisms:

$$
\begin{aligned}
a_{A B C} & : A \otimes(B \otimes C) \cong(A \otimes B) \otimes C \\
l_{A} & : K \otimes A \cong A \\
r_{A} & : A \otimes K \cong A
\end{aligned}
$$

such that

$\left.\left(A \otimes a_{B C D}\right) \circ a_{A(B \otimes C) D} \circ\left(a_{A B C} \otimes D\right)=a_{A B(C \otimes D}\right) \circ a_{(A \otimes B) C D}$

$\left(A \otimes l_{B}\right) \circ a_{A K B}=r_{A} \otimes B$ 
If there is a natural isomorphism $\tau_{B}^{A}: A \otimes B \cong B \otimes A$ such that $\tau_{A}^{B} \circ \tau_{B}^{A}=A \otimes B, \tau_{B \otimes C}^{A}=\left(B \otimes \tau_{C}^{A}\right) \circ\left(\tau_{B}^{A} \otimes C\right)$, then $\mathcal{C}$ is called a symmetric monoidal category.

A closed category is a symmetric monoidal category in which each functor $-\otimes A: \mathcal{C} \rightarrow \mathcal{C}$ has a specified right adjoint $[A,-]: \mathcal{C} \rightarrow \mathcal{C}([12]$, [18]).

Examples:

1) The category of sets and mappings.

2) The category of $R$-modules over a commutative ring $R$.

3) The category of chain complexes of $R$-modules and morphisms of degree 0 , with $R$ a commutative ring.

4) The category of sheaves of $\theta$-modules over a topological space, with $\theta$ a sheaf of commutative rings.

5) The category of coherent sheaves of modules over a scheme.

6 ) The category of all $R$-graded modules with morphisms of degree 0 ( $R$ is a commutative graded ring).

7) $(R, \sigma)$-Mod, with $R$ a commutative ring and $\sigma$ an idempotent kernel functor in $R$-Mod.

In what follows, $\mathcal{C}$ denotes a symmetric closed category with equalizers, co-equalizers and projective basic objec $K$. We denote by $\alpha_{M}$ and $\beta_{M}$ the unit and the co-unit, respectively, of the $\mathcal{C}$-adjuntion $M \otimes-\dashv[M,-]$ : $\mathcal{C} \rightarrow \mathcal{C}$ which exists for each object $M$ of $\mathcal{C}$.

1. An object $M$ of $\mathcal{C}$ is called profinite in $\mathcal{C}$ if the morphism $\left[M, \beta_{M}(K) \otimes M\right] \circ \alpha_{M}(\hat{M} \otimes M): \hat{M} \otimes M \rightarrow[M, M]=E(M)$ is an isomorphism, where $\hat{M}=[M, K]$. If, moreover, the factorization of $\beta_{M}(K): M \otimes \hat{M} \rightarrow K$ through the co-equalizer of the morphisms $\beta_{M}(M) \otimes \hat{M}$ and $M \otimes\left(\left[M, \beta_{M}(K) \circ\left(\beta_{M}(M) \otimes \hat{M}\right)\right] \circ \alpha_{M}(E(M) \otimes \hat{M})\right)$ : $M \otimes E(M) \otimes \hat{M} \rightarrow M \otimes \hat{M}$ is an isomorphism, we say that $M$ is a progenerator in $\mathcal{C}$.

2. A monoid in $\mathcal{C}$ is a triple $\mathbf{A}=\left(A, \eta_{A}, \mu_{A}\right)$ where $A$ is an object in $\mathcal{C}$ and $\mu_{A}: A \otimes A \rightarrow A, \eta_{A}: K \rightarrow A$ are morphisms in $\mathcal{C}$ such that $\mu_{A} \circ\left(A \otimes \eta_{A}\right)=A=\mu_{A} \circ\left(\eta_{A} \otimes A\right), \mu_{A} \circ\left(\mu_{A} \otimes A\right)=\mu_{A} \circ\left(A \otimes \mu_{A}\right)$. If $\mu_{A} \circ \tau_{A}^{A}=\mu_{A}$, then we will say that $\mathbf{A}$ is a commutative monoid. Given two monoids $\mathbf{A}=\left(A, \eta_{A}, \mu_{A}\right)$ and $\mathbf{B}=\left(B, \eta_{B}, \mu_{B}\right)$ in $C, f: A \rightarrow B$ is a monoid morphism if $\mu_{B} \circ(f \otimes f)=f \circ \mu_{A}$ and $f \circ \eta_{A}=\eta_{B}$.

A comonoid (cocommutative), $\mathbf{D}=\left(D, \varepsilon_{D}, \delta_{D}\right)$ is an object $D$ in $\mathcal{C}$ together with two morphisms $\varepsilon_{D}: D \rightarrow K, \delta_{D}: D \rightarrow D \otimes D$, such that $\left(\delta_{D} \otimes D\right) \circ \delta_{D}=\left(D \otimes \delta_{D}\right) \circ \delta_{D}$ and $\left(\varepsilon_{D} \otimes D\right) \circ \delta_{D}=1_{D}=(D \otimes$ $\left.\varepsilon_{D}\right) \circ \delta_{D}\left(\tau_{D}^{D} \circ \delta_{D}=\delta_{D}\right)$. If $\mathbf{D}=\left(D, \varepsilon_{D}, \delta_{D}\right)$ and $\mathbf{E}=\left(E, \varepsilon_{E}, \delta_{E}\right)$ are comonoids, $f: D \rightarrow E$ is a comonoid morphism if $(f \otimes f) \circ \delta_{D}=\delta_{E} \circ f$ and $\varepsilon_{E} \circ f=\varepsilon_{D}$. 
3. For a monoid $\mathbf{A}=\left(A, \eta_{A}, \mu_{A}\right)$ and a comonoid $\mathbf{D}=\left(D, \varepsilon_{D}, \delta_{D}\right)$ in $\mathcal{C}$, we denote by $\operatorname{Reg}(D, A)$ the group of invertible elements in $C(D, A)$ (morphisms in $C$ from $D$ to $A$ ) with the operation "convolution" given by: $f * g=\mu_{A} \circ(f \otimes g) \circ \delta_{D}$. The unit element is $\varepsilon_{D} \otimes \eta_{A}$.

Observe that $\operatorname{Reg}(D, A)$ is an abelian group when $\mathrm{D}$ is cocommutative and $\mathbf{A}$ is commutative.

4. Definition. Let $\Pi=\left(C, \eta_{C}, \mu_{C}\right)$ be a monoid and $\mathbf{C}=\left\langle C, \varepsilon_{C}, \delta_{C}\right)$ a comonoid in $\mathcal{C}$ and let $\lambda: C \rightarrow C$ be a morphism. Then $\mathbf{H}=(\mathbf{C}=$ $\left.\left(C, \varepsilon_{C}, \delta_{C}\right), \Pi=\left(C, \eta_{C}, \mu_{C}\right), \tau^{C}, \lambda\right)$ is a Hopf algebra in $\mathcal{C}$ with respect to the comonoid $\mathrm{C}$ if $\varepsilon_{C}$ and $\delta_{C}$ are monoid morphisms (equivalently, $\eta_{C}$ and $\mu_{C}$ are comonoid morphisms) and $\lambda$ is the inverse of $1_{C}: C \rightarrow C$ in $\operatorname{Reg}(C, C)$.

We say that $\mathbf{H}$ is a finite Hopf algebra if $C$ is profinite in $C$. if:

5. Definition. $\left(\mathbf{A}, \varphi_{A}\right)=\left(A, \eta_{A}, \mu_{A} ; \varphi_{A}\right)$ is a left $\mathbf{H}$-module monoid

i) $\mathbf{A}=\left(A, \eta_{A}, \mu_{A}\right)$ is a monoid in $C$.

ii) $\left(A, \varphi_{A}\right)$ is a left $\mathbf{H}$-module $\left(\varphi_{A} \circ\left(C \otimes \varphi_{A}\right)=\varphi_{A} \circ\left(\mu_{C} \otimes A\right), \varphi_{A} \circ\right.$ $\left.\left(\eta_{C} \otimes A\right)=A\right)$.

iii) $\eta_{A}, \mu_{A}$ are morphisms of left $\mathrm{H}$-modules $\left(\varphi_{A} \circ\left(C \otimes \eta_{A}\right)=\eta_{A} \otimes \varepsilon_{C}\right.$ and $\varphi_{A} \circ\left(C \otimes \mu_{A}\right)=\mu_{A} \circ \varphi_{A \otimes A}$, where $\varphi_{A \otimes A}=\left(\varphi_{A} \otimes \varphi_{A}\right) \circ(C \otimes$ $\left.\left.\tau_{A}^{C} \otimes A\right) \circ\left(\delta_{C} \otimes A \otimes A\right)\right)$.

We say that the action $\varphi_{A}$ of $\mathbf{H}$ in $\mathbf{A}$ is inner if there exists a morphism $f$ in $\operatorname{Reg}(C, A)$ such that $\varphi_{A}=\mu_{A} \circ\left(A \otimes\left(\mu_{A} \circ \tau_{A}^{A}\right)\right) \circ\left(f \otimes f^{-1} \otimes A\right) \circ$ $\left(\delta_{C} \otimes A\right): C \otimes A \rightarrow A$, where $f^{-1}$ is the convolution inverse of $f$.

6. Definition. If $\mathbf{H}$ is a cocommutative Hopf algebra and $\left(\mathbf{A}, \varphi_{A}\right)$ is a commutative $\mathbf{H}$-module monoid, then, we say that a morphism $\sigma$ in $\operatorname{Reg}(C \otimes C, A)$ is a 2-cocycle if $\partial_{1}(\sigma) * \partial_{3}(\sigma)=\partial_{2}(\sigma) * \partial_{4}(\sigma)$, where $\partial_{1}(\sigma)=\varphi_{A} \circ(C \otimes \sigma), \partial_{2}(\sigma)=\sigma \circ\left(\mu_{C} \otimes C\right), \partial_{3}(\sigma)=\sigma \circ\left(C \otimes \mu_{C}\right)$ and $\partial_{4}(\sigma)=\sigma \otimes \varepsilon_{C}$

Two 2-cocycles $\sigma$ and $\gamma$ are said to be cohomologous, written $\sigma \sim \gamma$, if there exists a morphism $v \in \operatorname{Reg}(C, A)$ such that $\sigma * \partial_{2}(v)=\partial_{1}(v) *$ $\partial_{3}(v) * \gamma$, where $\partial_{1}(v)=\varphi_{A} \circ(C \otimes v), \partial_{2}(v)=v \circ \mu_{C}$ and $\partial_{3}(v)=v \otimes \varepsilon_{C}$.

Trivially, " $\sim$ " is an equivalence relation.

The set of equivalence classes shall be called the second cohomology group of the cocommutative Hopf algebra $\mathbf{H}$ with the coefficients in the left $\mathbf{H}$-module monoid $\left(\mathbf{A}, \varphi_{A}\right)$, and will be denoted by $H^{2}(\mathbf{H}, \mathbf{A})$.

If $\sigma$ is a 2-cocycle in $\operatorname{Reg}(C \otimes C, A)$, then the morphism $\bar{\sigma}=\sigma * \partial_{1}(\pi) *$ $\partial_{2}\left(\pi^{-1}\right) * \partial_{3}(\pi)$ is a 2-cocycle in $\operatorname{Reg}(C \otimes C, A)$ cohomologous with $\sigma$ such that $\bar{\sigma} \circ\left(\eta_{C} \otimes C\right)=\varepsilon_{C} \otimes \eta_{A}=\bar{\sigma} \circ\left(C \otimes \eta_{C}\right)$, where $\pi=\sigma^{-1} \circ\left(C \otimes \eta_{C}\right)$ is a morphism in $\operatorname{Reg}(C, A)$ with inverse $\pi^{-1}=\sigma \circ\left(C \otimes \eta_{C}\right)$. Moreover, if 
$\gamma$ is cohomologous with $\sigma$, then there exists a morphism $\bar{\vartheta} \in \operatorname{Reg}(C, A)$ such that $\bar{\vartheta} \circ \eta_{C}=\eta_{A}$ and $\bar{\sigma} * \partial_{2}(\bar{\vartheta})=\partial_{1}(\bar{\vartheta}) * \partial_{3}(\bar{\vartheta}) * \bar{\gamma}$.

Remark. Let $\mathcal{C}$ the category of $K$-modules over a field. In this case, $H^{2}(\mathbf{H}, \mathbf{A})$ is the second cohomology group of the Sweedler's complex $\left\{\operatorname{Reg}(\stackrel{q}{\otimes} C, A) ; \Delta_{q}\right\}_{q \geq 0}$

$$
\begin{aligned}
\operatorname{Reg}(K, A) \stackrel{\Delta_{0}}{\longrightarrow} \operatorname{Reg}(C, A) \stackrel{\Delta_{1}}{\longrightarrow} \ldots \stackrel{\stackrel{\Delta_{q-1}}{\longrightarrow} \operatorname{Reg}(\otimes C, A)}{ } & \stackrel{\Delta_{q}}{\longrightarrow} \operatorname{Reg}(\stackrel{q+1}{\otimes} C, A) \stackrel{\Delta_{q+1}}{\longrightarrow} \ldots
\end{aligned}
$$

where $\Delta_{q}:=\partial_{1} * \partial_{2}^{-1} * \cdots * \partial_{q+2}^{(-1)^{q \cdot 1}}$ and for each $f \in \operatorname{Reg}(\otimes C, A)$,

$$
\begin{aligned}
& \partial_{1}(f)=\varphi_{A} \circ(C \otimes f) \\
& \vdots \\
& \partial_{i}(f)=f \circ\left(C \otimes i-2 \otimes C \otimes \mu_{C} \otimes C \otimes{ }^{q-i+1} \otimes C\right) \\
& \vdots \\
& \partial_{q+2}(f)=f \otimes \varepsilon_{C}
\end{aligned}
$$

([21]).

7. Definition. $\left(\mathbf{B}, \rho_{B}\right)=\left(B, \eta_{B}, \mu_{B} ; \rho_{B}\right)$ is a right $\mathbf{H}$-comodule monoid if:

i) $\mathbf{B}=\left(B, \eta_{B}, \mu_{B}\right)$ is a monoid in $\mathcal{C}$

ii) $\left(B, \rho_{B}\right)$ is a right $\mathrm{H}-$ comodule $\left(\left(\rho_{B} \otimes C\right) \circ \rho_{B}=\left(B \otimes \delta_{C}\right) \circ \rho_{B}:(B \otimes\right.$ $\left.\varepsilon_{C}\right) \circ \rho_{B}=B$ )

iii) $\rho_{B}: B \rightarrow B \otimes C$ is a monoid morphism from $\left(B, \eta_{B}, \mu_{B}\right)$ to the product monoid $\mathrm{B} \Pi=\left(B \otimes C, \eta_{B} \otimes \eta_{C},\left(\mu_{B} \otimes \mu_{C}\right) \circ\left(B \otimes \tau_{B}^{C} \otimes C\right)\right)$ (that is, $\rho_{B} \circ \eta_{B}=\eta_{B} \otimes \eta_{C}$ and $\rho_{B} \circ \mu_{B}=\left(\mu_{B} \otimes \mu_{C}\right) \circ\left(B \otimes \tau_{B}^{C} \otimes\right.$ C) $\left.\circ\left(\rho_{B} \otimes \rho_{B}\right)\right)$.

From now on we assume that $H$ is a finite cocommutative and commutative Hopf algebra.

8. Definition. A right $\mathbf{H}$-comodule monoid $\left(\mathrm{B}, \rho_{B}\right)$ is said to be a Galois $\mathbf{H}$-object if and only if:

i) The morphism $\gamma_{B}:=\left(\mu_{B} \otimes C\right) \circ\left(B \otimes \rho_{B}\right): B \otimes B \rightarrow B \otimes C$ is an isomorphism.

ii) $B$ is a progenerator in $C$.

For example, in the case of $(R, \sigma)$-Mod, a commutative $\mathbf{H}$-comodule monoid is a couple ( $\left.\mathrm{B}, \rho_{B}\right)$, where $B$ is a commutative $(R, \sigma)$-algebra and $\rho_{B}: B \rightarrow B \perp H:=Q_{\sigma}\left(B \otimes_{R} H\right)$ is a morphism of algebras and it defines a right $\mathbf{H}$-comodule structure over $B$. 
$\left(\mathbf{B}, \rho_{B}\right)$ is a Galois $\mathbf{H}$-object if and only if $B$ is a $(R, \sigma)$-progenerator and the mapping $\rho_{B}^{\hat{H}}: \underset{\sigma}{B} \hat{H} \rightarrow \operatorname{Hom}(B, B)$ arising from the left $\underset{\sigma}{B} \hat{H}$ module structure on $B$ is an isomorphism ( $[15,(1.3 .17)])$.

If a Galois $\mathbf{H}$-object is isomorphic to $\mathbf{H}$ as an $\mathbf{H}$-comodule then we say that it has a normal basis.

If $\mathbf{B}_{1}$ and $\mathbf{B}_{2}$ are Galois $\mathbf{H}$-objects, $f: B_{1} \rightarrow B_{2}$ is a morphism of Galois $\mathbf{H}$-objects if it is a morphism of $\mathbf{H}$-comodules $\left(\rho_{B_{2}} \circ f=(f \otimes C) \circ\right.$ $\left.\rho_{B_{1}}\right)$ and of monoids.

If $\left(\mathbf{A}, \rho_{A}\right)$ and $\left(\mathbf{B}, \rho_{B}\right)$ are $\mathbf{H}$-comodule monoids, then $\mathbf{A} \circ \mathbf{B}$, defined by the following equalizer diagram

$$
A \circ B \stackrel{i_{A B}}{\longrightarrow} A \otimes B \underset{\partial_{A B}^{2}}{\stackrel{\partial_{A B}^{2}}{\rightrightarrows}} A \otimes B \otimes C
$$

where

$$
\begin{aligned}
& \partial_{A B}^{1}=\left(A \otimes \tau_{B}^{C}\right) \circ\left(\rho_{A} \otimes B\right), \text { and } \\
& \partial_{A B}^{2}=A \otimes \rho_{B}
\end{aligned}
$$

is an $\mathbf{H}$-comodule monoid to be denoted by $\left(\mathbf{A} \circ \mathbf{B}, \rho_{A B}\right)$.

If moreover $\left(\mathbf{A}, \rho_{A}\right)$ and $\left(\mathbf{B}, \rho_{B}\right)$ are Galois $\mathbf{H}$-objects, then $(\mathbf{A} \circ$ $\left.\mathbf{B}, \rho_{A B}\right)$ is also a Galois $\mathbf{H}$-object, where $\rho_{A B}$ is the factorization of the morphism $\partial_{A B}^{1} \circ i_{A B}$ (or $\partial_{A B}^{2} \circ i_{A B}$ ) through the equalizer $i_{A B} \otimes C$.

The set of isomorphism classes of Galois $\mathbf{H}$-objects (with a normal basis), with the operation induced by the one given above, is an abelian group to be denoted by $\mathrm{Gal}_{\mathcal{C}}(\mathbf{H})\left(\mathrm{N}_{\mathcal{C}}(\mathbf{H})\right)$. The unit element is the class of $\left(\Pi, \delta_{C}\right)$ and the opposite of $\left[\left(\mathbf{B}, \rho_{B}\right)\right]$ is $\left[\left(\mathbf{B}^{\circ \mathrm{P}},(B \otimes \lambda) \circ \rho_{B}\right)\right]$ where $\mathbf{B}^{\circ \mathrm{P}}=\left(B, \eta_{B}, \mu_{B} \circ \tau_{B}^{B}\right)$.

Remark. In the case of a finitely generated projective, commutative and cocommutative Hopf algebra $\mathbf{H}$ over a commutative ring $R, \mathrm{Gal}_{C}(\mathbf{H})$ is the group of Galois $\mathbf{H}$-objects in the sense of $\mathrm{S}$. Chase and $\mathrm{M}$. Sweedler in $[\mathbf{9}]$.

9. Proposition. If $\left[\left(\mathbf{B}, \rho_{B}\right)\right] \in \mathbf{N}_{C}(\mathbf{H})$, then, there is a 2-cocycle $\sigma$ in $\operatorname{Reg}(C \otimes C, K)$ satisfying $\sigma \circ\left(\eta_{C} \otimes C\right)=\varepsilon_{C}=\sigma \circ\left(C \otimes \eta_{C}\right)$.

\section{Proof:}

Let $\left(\mathbf{B}, \rho_{B}\right)$ a Galois $\mathbf{H}$-object with a normal basis. Then we have an isomorphism $\gamma_{B}: B \otimes B \rightarrow B \otimes C$ and an $\mathbf{H}$-comodule isomorphism $r: C \rightarrow B$. Therefore the morphism of $\mathbf{H}$-comodules $f=\left(\varepsilon_{C} \otimes B\right) \circ$ $\left(r^{-1} \otimes r\right) \circ\left(\eta_{B} \otimes C\right): C \rightarrow B$ is in $\operatorname{Reg}(C, B)$ with inverse

$$
f^{-1}=\mu_{B} \circ\left(B \otimes \varepsilon_{C} \otimes B\right) \circ\left(B \otimes r^{-1} \otimes r\right) \circ\left(\gamma_{B}^{-1} \otimes \eta_{C}\right) \circ\left(\eta_{B} \otimes C\right)
$$


and satisfying $f \circ \eta_{C}=\eta_{B}$.

Indeed:

$$
\begin{aligned}
f * f^{-1} & =\mu_{B} \circ\left(\varepsilon_{C} \otimes B \otimes \varepsilon_{C} \otimes B\right) \circ\left(r^{-1} \otimes B \otimes r^{-1} \otimes r\right) \circ \\
& \circ\left(\eta_{B} \otimes \gamma_{B}^{-1} \otimes \eta_{C}\right) \circ(r \otimes C) \circ \delta_{C}= \\
& =\mu_{B} \circ\left(\varepsilon_{C} \otimes B \otimes \varepsilon_{C} \otimes B\right) \circ\left(r^{-1} \otimes B \otimes r^{-1} \otimes r\right) \circ \\
& \circ\left(B \otimes\left[\gamma_{B}^{-1} \circ \gamma_{B}\right] \otimes C\right) \circ\left(\eta_{B} \otimes \eta_{B} \otimes B \otimes \eta_{C}\right) \circ r= \\
& \left.=\left(\varepsilon_{C} \otimes B \otimes \varepsilon_{C}\right) \circ\left(r^{-1} \otimes r \otimes C\right) \circ\left(\rho_{B} \circ \eta_{B}\right] \otimes C\right)= \\
& =\varepsilon_{C} \otimes \eta_{B} \\
f^{-1} * f & =\mu_{B} \circ\left(B \otimes\left[\varepsilon_{C} \circ r^{-1}\right] \otimes B\right) \circ\left(\gamma_{B}^{-1} \otimes r\right) \circ\left(\eta_{B} \otimes \delta_{C}\right)= \\
& =\mu_{B} \circ\left(B \otimes\left[\varepsilon_{C} \circ r^{-1}\right] \otimes r\right) \circ\left(B \otimes \rho_{B}\right) \circ \gamma_{B}^{-1} \circ\left(\eta_{B} \otimes C\right)= \\
& =\mu_{B} \circ\left(B \otimes \varepsilon_{C} \otimes r\right) \circ\left(B \otimes \delta_{C}\right) \circ\left(B \otimes r^{-1}\right) \circ \gamma_{B}^{-1} \circ\left(\eta_{B} \otimes C\right)= \\
& =\left(B \otimes \varepsilon_{C}\right) \circ \gamma_{B} \circ \gamma_{B}^{-1} \circ\left(\eta_{B} \otimes C\right)= \\
& =\varepsilon_{C} \otimes \eta_{B}
\end{aligned}
$$

because $r$ is an $\mathrm{H}$-comodule isomorphism and the equalities:

$\left(\mu_{B} \otimes B\right) \circ\left(B \otimes \gamma_{B}^{-1}\right)=\gamma_{B}^{-1} \circ\left(\mu_{B} \otimes C\right)$ $\left(\varepsilon_{C} \otimes B\right) \circ\left(r^{-1} \otimes r\right) \circ\left(\eta_{B} \otimes \eta_{C}\right)=\left(\varepsilon_{C} \otimes B\right) \circ\left(r^{-1} \otimes r\right) \circ \rho_{B} \circ \eta_{B}=\eta_{B}$ $\left(\gamma_{B}^{-1} \otimes C\right) \circ\left(B \otimes \delta_{C}\right)=\left(B \otimes \rho_{B}\right) \circ \gamma_{B}^{-1}$

Trivially, $f$ is a morphism of $\mathbf{H}$-comodules and $f \circ \eta_{C}=\eta_{B}$.

The morphism $\sigma_{f}=\left(\mu_{B} \circ(f \otimes f)\right) *\left(f^{-1} \otimes \mu_{C}\right): C \otimes C \rightarrow B$ factors through the equalizer

$$
K \stackrel{\eta_{B}}{\rightarrow} B \underset{B \otimes \eta_{C}}{\stackrel{\rho_{B}}{\rightrightarrows}} B \otimes C
$$

because $\mathbf{H}$ is a Hopf algebra, $\left(\mathbf{B}, \rho_{B}\right)$ an $\mathbf{H}$-comodule monoid, $f$ a morphism of $\mathbf{H}$-comodules and the equality $\rho_{B} \circ f^{-1}=\left(f^{-1} \otimes \lambda\right) \circ \tau_{C}^{C} \circ \delta_{C}$ $([14,(2.3)])$.

Moreover, the factorization, $\bar{\sigma}_{f}$, of $\sigma_{f}$ is in $\operatorname{Reg}(C \otimes C, K)$ with inverse the factorization of the morphism $\sigma_{f}^{-1}=\left(f \circ \mu_{C}\right) *\left(\mu_{B} \circ \tau_{B}^{B} \circ\left(f^{-1} \otimes f^{-1}\right)\right)$ : $C \otimes C \rightarrow B$ through the equalizer $\eta_{B}$. 
The morphism $\bar{\sigma}_{f}: C \otimes C \rightarrow K$ is a 2-cocycle. Indeed:

$$
\begin{aligned}
\eta_{B} & \circ\left(\partial_{1}\left(\bar{\sigma}_{f}\right) * \partial_{3}\left(\bar{\sigma}_{f}\right)\right)= \\
& =\left(f * f^{-1}\right) \circ\left(\bar{\sigma}_{f} \otimes \mu_{C}\right) \circ\left(C \otimes \tau_{C}^{C} \otimes C\right) \circ\left(\delta_{C} \otimes \delta_{C}\right) \circ \\
& \circ\left(C \otimes \bar{\sigma}_{f} \otimes \mu_{C}\right) \circ\left(C \otimes C \otimes \tau_{C}^{C} \otimes C\right) \circ\left(C \otimes \delta_{C} \otimes \delta_{C}\right)= \\
& =\mu_{B} \circ\left(B \otimes f^{-1}\right) \circ \rho_{B} \circ f \circ\left(\bar{\sigma}_{f} \otimes \mu_{C}\right) \circ\left(C \otimes \tau_{C}^{C} \otimes C\right) \circ\left(\delta_{C} \otimes \delta_{C}\right) \circ \\
& \circ\left(C \otimes \bar{\sigma}_{f} \otimes \mu_{C}\right) \circ\left(C \otimes C \otimes \tau_{C}^{C} \otimes C\right) \circ\left(C \otimes \delta_{C} \otimes \delta_{C}\right)= \\
& =\mu_{B} \circ\left(B \otimes f^{-1}\right) \circ \rho_{B} \circ \mu_{B} \circ(f \otimes f) \circ\left(C \otimes \bar{\sigma}_{f} \otimes \mu_{C}\right) \circ \\
& \circ\left(C \otimes C \otimes \tau_{C}^{C} \otimes C\right) \circ\left(C \otimes \delta_{C} \otimes \delta_{C}\right)= \\
& =\mu_{B} \circ\left(B \otimes f^{-1}\right) \circ \rho_{B} \circ \mu_{B} \circ\left(\mu_{B} \otimes C\right) \circ(f \otimes f \otimes f)= \\
& =\mu_{B} \circ\left(B \otimes f^{-1}\right) \circ \rho_{B} \circ f \circ\left(\bar{\sigma}_{f} \otimes \mu_{C}\right) \circ\left(C \otimes \tau_{C}^{C} \otimes C\right) \circ\left(\delta_{C} \otimes \delta_{C}\right) \circ \\
& \circ\left(\bar{\sigma}_{f} \otimes \mu_{C} \otimes C\right) \circ\left(C \otimes \tau_{C}^{C} \otimes C \otimes C\right) \circ\left(\delta_{C} \otimes \delta_{C} \otimes C\right)= \\
& =\left(f * f^{-1}\right) \circ\left(\bar{\sigma}_{f} \otimes \mu_{C}\right) \circ\left(C \otimes \tau_{C}^{C} \otimes C\right) \circ\left(\delta_{C} \otimes \delta_{C}\right) \circ \\
& \circ\left(\bar{\sigma}_{f} \otimes \mu_{C} \otimes C\right) \circ\left(C \otimes \tau_{C}^{C} \otimes C \otimes C\right) \circ\left(\delta_{C} \otimes \delta_{C} \otimes C\right)= \\
& =\eta_{B} \circ\left(\partial_{2}\left(\bar{\sigma}_{f}\right) * \partial_{4}\left(\bar{\sigma}_{f}\right)\right)
\end{aligned}
$$

because $f$ is a morphism of $\mathbf{H}$-comodules, $\mathbf{H}$ a cocommutative Hopf algebra and the equality

$$
f \circ\left(\bar{\sigma}_{f} \otimes \mu_{C}\right) \circ\left(C \otimes \tau_{C}^{C} \otimes C\right) \circ\left(\delta_{C} \otimes \delta_{C}\right)=\mu_{B} \circ(f \otimes f)
$$

and then, since $\eta_{B}$ is a monomorphism, $\bar{\sigma}_{f}$ is a 2-cocycle.

Trivially, $\bar{\sigma}_{f} \circ\left(\eta_{C} \otimes C\right)=\varepsilon_{C}=\bar{\sigma}_{f} \circ\left(C \otimes \eta_{C}\right)$.

Remark. If $\left[\left(\mathbf{B}_{1}, \rho_{B_{1}}\right)\right]=\left[\left(\mathbf{B}_{2}, \rho_{B_{2}}\right)\right] \in \mathbf{N}_{C}(\mathbf{H})$ then there is an isomorphism of $\mathbf{H}$-comodule monoids $h: B_{1} \rightarrow B_{2}$. Clearly, $\overline{\sigma_{f_{1}}}=\overline{\sigma_{h \circ f_{1}}}$. (Notice that $h \circ f_{1} \in \operatorname{Reg}\left(C, B_{2}\right)$ with inverse $h \circ f_{1}^{-1}$ ). Moreover, $\overline{\sigma_{f_{1}}} \sim \overline{\sigma_{f_{2}}}$.

Indeed:

The morphism $e=\left(h \circ f_{1}\right) * f_{2}^{-1}: C \rightarrow B_{2}$ factors through the equalizer $\eta_{B_{2}}$ :

$$
\begin{aligned}
& \rho_{B_{2}} \circ e=\left(\mu_{B_{2}} \otimes \mu_{C}\right) \circ(\left.\left(h \circ f_{1}\right) \otimes \tau_{B_{2}}^{C} \otimes \lambda\right) \circ\left(\delta_{C} \otimes f_{2}^{-1} \otimes C\right) \circ \\
& \circ\left(C \otimes\left(\tau_{C}^{C} \circ \delta_{C}\right)\right) \circ \delta_{C}=\left(B_{2} \otimes \eta_{C}\right) \circ e
\end{aligned}
$$

and then, there cxists a morphism $\bar{e}: C \rightarrow K$ such that $\eta_{B_{2}} \circ \bar{e}=e$. Clearly, $\bar{e} \circ \eta_{C}=K$. Moreover, $\bar{e}$ is in $\operatorname{Reg}(C, K)$ with inverse $\bar{e}^{-1}$, the factorization of $e^{-1}=f_{2} *\left(h \circ f_{1}^{-1}\right)$ throught the equalizer $\eta_{B_{2}}$. 
We also have that:

$$
\begin{aligned}
\eta_{B_{2}} & \circ\left(\overline{\sigma_{f_{2}}} * \partial_{2}(\bar{e})\right)= \\
& =\mu_{B_{2}} \circ\left(\eta_{B_{2}} \otimes \eta_{B_{2}}\right) \circ\left(\overline{\sigma_{h \circ f}} * \partial_{2}(\bar{e})\right)= \\
& =\mu_{B_{2}} \circ\left(\mu_{B_{2}} \otimes \mu_{B_{2}}\right) \circ\left[\left(h \circ f_{1}\right) \otimes\left(h \circ f_{1}\right) \otimes\left(\left(h \circ f_{1}^{-1}\right) *\left(h \circ f_{1}\right)\right) \otimes\right. \\
& \left.\otimes f_{2}^{-1}\right] \circ\left(C \otimes C \otimes \delta_{C}\right) \circ\left(C \otimes C \otimes \mu_{C}\right) \circ\left(C \otimes \tau_{C}^{C} \otimes C\right) \circ\left(\delta_{C} \otimes \delta_{C}\right)= \\
& =\mu_{B_{2}} \circ\left(\mu_{B_{2}} \otimes B_{2}\right) \circ\left(\mu_{B_{2}} \otimes\left(\mu_{B_{2}} \circ \tau_{B_{2}}^{B_{2}}\right) \otimes \mu_{B_{2}}\right) \circ \\
& \circ\left(\left(h \circ f_{1}\right) \otimes\left(h \circ f_{1}\right) \otimes\left(f_{2}^{-1} * f_{2}\right) \otimes f_{2}^{-1} \otimes f_{2} \otimes f_{2}^{-1}\right) \circ \\
& \circ\left(C \otimes C \otimes C \otimes \delta_{C} \otimes \mu_{C}\right) \circ\left(C \otimes C \otimes C \otimes \tau_{C}^{C} \otimes C\right) \circ \\
& \circ\left(C \otimes C \otimes \delta_{C} \otimes \delta_{C}\right) \circ\left(C \otimes \tau_{C}^{C} \otimes C\right) \circ\left(\delta_{C} \otimes \delta_{C}\right)= \\
& =\mu_{B_{2}} \circ\left(\mu_{B_{2}} \otimes B_{2}\right) \circ\left(\left(\eta_{B_{2}} \circ \bar{e}\right) \otimes\left(\eta_{B_{2}} \circ \bar{e}\right) \otimes\left(\eta_{B_{2}} \circ \overline{\sigma_{f_{2}}}\right)\right) \circ \\
& \circ\left(C \otimes \tau_{C}^{C} \otimes C\right) \circ\left(\delta_{C} \otimes \delta_{C}\right)= \\
& =\eta_{B_{2}} \circ\left(\partial_{1}(\bar{e}) * \partial_{3}(\bar{e}) * \overline{\sigma_{f_{2}}}\right)
\end{aligned}
$$

and then, since $\eta_{B_{2}}$ is a monomorphism, $\overline{\sigma_{f_{1}}} \sim \overline{\sigma_{f_{2}}}$.

10. Proposition. If $\sigma$ is a 2-cocycle in $\operatorname{Reg}(C \otimes C, K)$ such that $\sigma \circ\left(\eta_{C} \otimes C\right)=\varepsilon_{C}=\sigma \circ\left(C \otimes \eta_{C}\right)$, then $\left(\mathrm{C}_{\sigma}=\left(C, \eta_{C}, \mu_{C_{\sigma}}=\left(\sigma \otimes \mu_{C}\right) \circ\right.\right.$ $\left.\left.\left(C \otimes \tau_{C}^{C} \otimes C\right) \circ\left(\delta_{C} \otimes \delta_{C}\right)\right) ; \delta_{C}\right)$ is a Galois $\mathbf{H}$-object with a normal basis.

\section{Proof:}

Trivially, $\left(\mathbf{C}_{a}, \delta_{C}\right)$ is an $\mathbf{H}$-comodule monoid.

The morphism $\gamma_{C_{\sigma}}=\left(\mu_{C_{\sigma}} \otimes C\right) \circ\left(C \otimes \delta_{C}\right): C \otimes C \rightarrow C \otimes C$ is an isomorphism with inverse

$$
\begin{array}{r}
\gamma_{C_{\sigma}}^{-1}=\left(\mu_{C} \otimes C\right) \circ\left(C \otimes \sigma^{-1} \otimes \lambda \otimes C\right) \circ\left(C \otimes \mu_{C} \otimes C \otimes \delta_{C}\right) \circ\left(\delta_{C} \otimes \lambda \otimes C \otimes C\right) \circ \\
\circ\left(C \otimes \delta_{C} \otimes C\right) \circ\left(C \otimes \delta_{C}\right)
\end{array}
$$

Indeed:

$$
\begin{aligned}
& \gamma_{C_{\sigma}}^{-1} \circ \gamma_{C_{\sigma}}= \\
& \quad=\left(\mu_{C} \otimes C\right) \circ\left(\mu_{C} \otimes \sigma^{-1} \otimes \lambda \otimes C\right) \circ\left(C \otimes C \otimes \mu_{C} \otimes C \otimes \delta_{C}\right) \circ \\
& \quad \circ\left(C \otimes C \otimes \mu_{C} \otimes \lambda \otimes C \otimes C\right) \circ\left(C \otimes \tau_{C}^{C} \otimes C \otimes \delta_{C} \otimes C\right) \circ \\
& \quad \circ\left(\sigma \otimes \delta_{C} \otimes \delta_{C} \otimes \delta_{C}\right) \circ\left(C \otimes \tau_{C}^{C} \otimes C \otimes C\right) \circ\left(\delta_{C} \otimes \delta_{C} \otimes C\right) \circ\left(C \otimes \delta_{C}\right)= \\
& \quad=\left(\mu_{C} \otimes C\right) \circ\left(\mu_{C} \otimes \sigma^{-1} \otimes \lambda \otimes C\right) \circ\left(C \otimes C \otimes \mu_{C} \otimes \delta_{C} \otimes C\right) \circ \\
& \circ\left(C \otimes \tau_{C}^{C} \otimes\left[\mu_{C} \circ(C \otimes \lambda) \circ \delta_{C}\right) \otimes \delta_{C}\right) \circ\left(C \otimes C \otimes \delta_{C} \otimes C\right) \circ \\
& \quad \circ\left(\sigma \otimes \delta_{C} \otimes \delta_{C}\right) \circ\left(C \otimes \tau_{C}^{C} \otimes C\right) \circ\left(\delta_{C} \otimes \delta_{C}\right)=
\end{aligned}
$$


$=\left(\mu_{C} \otimes C\right) \circ\left(\mu_{C} \otimes \sigma^{-1} \otimes \lambda \otimes C\right) \circ\left(C \otimes \tau_{C}^{C} \otimes\left[\tau_{C}^{C} \circ \delta_{C}\right] \otimes C\right) \circ$ $\circ\left(\sigma \otimes \delta_{C} \otimes C \otimes \delta_{C}\right) \circ\left(C \otimes \tau_{C}^{C} \otimes \delta_{C}\right) \circ\left(\delta_{C} \otimes \delta_{C}\right)=$ $=\left(\mu_{C} \otimes C\right) \circ\left(C \otimes\left[\mu_{C} \circ(C \otimes \lambda) \circ \delta_{C}\right] \otimes C\right) \circ$

$\circ\left(\sigma \otimes C \otimes C \otimes \sigma^{-1} \otimes C\right) \circ\left(C \otimes C \otimes C \otimes \tau_{C}^{C} \otimes C \otimes C\right) \circ$ $\circ\left(C \otimes C \otimes \delta_{C} \otimes \delta_{C} \otimes C\right) \circ\left(C \otimes \tau_{C}^{C} \otimes \delta_{C}\right) \circ\left(\delta_{C} \otimes \delta_{C}\right)=$ $=\left(\sigma \otimes C \otimes \sigma^{-1} \otimes C\right) \circ\left(C \otimes C \otimes\left[\tau_{C}^{C} \circ \delta_{C}\right] \otimes \delta_{C}\right) \circ$ $\circ\left(C \otimes \tau_{C}^{C} \otimes C\right) \circ\left(\delta_{C} \otimes \delta_{C}\right)=$ $=\left(\left[\sigma * \sigma^{-1}\right] \otimes C \otimes C\right) \circ\left(C \otimes \tau_{C}^{C} \otimes C\right) \circ\left(\delta_{C} \otimes \delta_{C}\right)=$ $=C \otimes C$ $\gamma_{C_{\sigma}} \circ \gamma_{C_{\sigma}}^{-1}=$

$=\left(\sigma \otimes \mu_{C} \otimes C\right) \circ\left(\mu_{C} \otimes \tau_{C}^{C} \otimes C \otimes C\right) \circ\left(C \otimes C \otimes \mu_{C} \otimes \delta_{C} \otimes C\right) \circ$ $\circ\left(C \otimes \tau_{C}^{C} \otimes C \otimes \delta_{C}\right) \circ\left(\delta_{C} \otimes \sigma^{-1} \otimes \delta_{C} \otimes C\right) \circ\left(C \otimes \mu_{C} \otimes C \otimes \lambda \otimes C\right) \circ$ $\circ\left(C \otimes C \otimes \lambda \otimes C \otimes \delta_{C}\right) \circ\left(\delta_{C} \otimes \delta_{C} \otimes C\right) \circ\left(C \otimes \delta_{C}\right)=$ $=\left(\sigma \otimes \mu_{C} \otimes C\right) \circ\left(C \otimes \tau_{C}^{C} \otimes C \otimes C\right) \circ\left(\mu_{C} \otimes \mu_{C} \otimes\left[\tau_{C}^{C} \circ \delta_{C}\right] \otimes C\right) \circ$ $\circ\left(C \otimes \tau_{C}^{C} \otimes C \otimes \delta_{C}\right) \circ\left(\delta_{C} \otimes \sigma^{-1} \otimes\left[(\lambda \otimes \lambda) \circ \tau_{C}^{C} \circ \delta_{C}\right] \otimes C\right) \circ$ $\circ\left(C \otimes \mu_{C} \otimes C \otimes C \otimes C\right) \circ\left(\delta_{C} \otimes \lambda \otimes C \otimes \delta_{C}\right) \circ$ $\circ\left(C \otimes \delta_{C} \otimes C\right) \circ\left(C \otimes \delta_{C}\right)=$ $=(\sigma \otimes C \otimes C) \circ\left(C \otimes \tau_{C}^{C} \otimes C\right) \circ\left(\mu_{C} \otimes \mu_{C} \otimes \delta_{C}\right) \circ$ $\circ\left(C \otimes \tau_{C}^{C} \otimes\left[\mu_{C} \circ(\lambda \otimes C) \circ \delta_{C}\right] \otimes C\right) \circ\left(\delta_{C} \otimes \sigma^{-1} \otimes \lambda \otimes \delta_{C}\right) \circ$ $\circ\left(C \otimes \mu_{C} \otimes C \otimes \delta_{C}\right) \circ\left(\delta_{C} \otimes \lambda \otimes C \otimes C\right) \circ\left(C \otimes \delta_{C} \otimes C\right) \circ\left(C \otimes \delta_{C}\right)=$ $=(\sigma \otimes C \otimes C) \circ\left(\mu_{C} \otimes r_{C}^{C} \otimes C\right) \circ\left(C \otimes \tau_{C}^{C} \otimes \delta_{C}\right) \circ$ $\circ\left(\left[\tau_{C}^{C} \circ \delta_{C}\right] \otimes \sigma^{-1} \otimes \lambda \otimes C\right) \circ\left(C \otimes \mu_{C} \otimes C \otimes C \otimes C\right) \circ$ $\circ(C \otimes C \otimes \lambda \otimes C \otimes C \otimes C) \circ\left(\delta_{C} \otimes \delta_{C} \otimes C \otimes C\right) \circ$ $\circ\left(C \otimes\left[\tau_{C}^{C} \circ \delta_{C}\right] \otimes C\right) \circ\left(C \otimes \delta_{C}\right)=$ $=(C \otimes \sigma \otimes C) \circ\left(C \otimes C \otimes \sigma^{-1} \otimes \delta_{C}\right) \circ\left(C \otimes\left[\delta_{C} \circ \mu_{C}\right] \otimes C \otimes C\right) \circ$ $\circ(C \otimes C \otimes \lambda \otimes C \otimes C) \circ\left(\delta_{C} \otimes \delta_{C} \otimes C\right) \circ\left(C \otimes \delta_{C}\right)=$ $=\left(C \otimes \sigma \otimes \sigma^{-1} \otimes C\right) \circ\left(C \otimes C \otimes \tau_{C}^{C} \otimes C \otimes C\right) \circ$ $\circ\left(C \otimes \delta_{C} \otimes \delta_{C} \otimes C\right) \circ\left(C \otimes \mu_{C} \otimes \delta_{C}\right) \circ\left(\delta_{C} \otimes \lambda \otimes C\right) \circ\left(C \otimes \delta_{C}\right)=$ $=\left(C \otimes\left[\sigma * \sigma^{-1}\right] \otimes C\right) \circ\left(C \otimes \mu_{C} \otimes \delta_{C}\right) \circ\left(\delta_{C} \otimes \lambda \otimes C\right) \circ\left(C \otimes \delta_{C}\right)=$ $=C \otimes C$

and thus $\left(C_{\sigma}, \delta_{C}\right)$ is a Galois $\mathbf{H}$-object with a normal basis.

Remark. If $\sigma$ and $\gamma$ are two 2-cocycles such in Proposition 10 and cohomologous, then $(\ddot{e} \otimes C) \circ \delta_{C}:\left(\mathrm{C}_{\sigma}, \delta_{C}\right) \rightarrow\left(\mathrm{C}_{\gamma}, \delta_{C}\right)$ is an isomorphism 
of $\mathrm{H}$-comodule monoids where $\bar{e}$ is the morphism which exists because $\sigma \sim \gamma$, and thus, $\left[\left(\mathbf{C}_{\sigma}, \delta_{C}\right)\right]=\left[\left(\mathbf{C}_{\gamma}, \delta_{C}\right)\right]$ in $\mathbf{N}_{C}(\mathbf{H})$.

11. Theorem. There is an isomorphism of abelian groups $F$ : $\mathbf{N}_{\mathcal{C}}(\mathbf{H}) \rightarrow H^{2}(\mathrm{H}, K)$ defined by $F\left(\left[\left(\mathbf{B}, \rho_{B}\right)\right]\right)=\left[\vec{\sigma}_{f}\right]$ with inverse $G([\sigma])=\left[\left(\mathrm{C}_{\sigma}, \delta_{C}\right)\right]$.

\section{Proof:}

The morphisms $F$ and $G$ are well defined by Propositions 9 and 10 . Moreover, the morphism of $\mathrm{H}$-comodules $f:\left(\mathrm{C}_{\bar{\sigma}_{f}}=\left(C, \eta_{C}, \mu_{C_{\sigma_{f}}}\right) ; \delta_{C}\right) \rightarrow$ ( $\left.\mathbf{B}=\left(B, \eta_{B}, \mu_{B}\right) ; \rho_{B}\right)$ is a morphism of monoids and therefore it is an isomorphism $([13,(4.3 .9)])$ and $\left[\left(\mathbf{B}, \rho_{B}\right)\right]=\left[\left(C_{\bar{\sigma}_{f}}, \delta_{C}\right)\right]$ in $\mathbf{N}_{C}(\mathbf{H})$.

If $[\sigma] \in H^{2}(\mathbf{H}, K)$ then the 2-cocycle $\gamma$ defined from $C_{\sigma}$ (Proposition 9) equals to $\sigma$ :

$$
\begin{aligned}
\eta_{C} \circ \gamma & =\mu_{C_{\sigma}} \circ\left(\mu_{C_{\sigma}} \otimes\left[\left(C \otimes \varepsilon_{C}\right) \circ \gamma_{C_{\sigma}}^{-1} \circ\left(\eta_{C} \otimes C\right)\right]\right) \circ\left(C \otimes C \otimes \mu_{C}\right) \circ \\
& \circ\left(C \otimes \tau_{C}^{C} \otimes C\right) \circ\left(\delta_{C} \otimes \delta_{C}\right)= \\
& =\left(\sigma \otimes \mu_{C}\right) \circ\left(C \otimes \tau_{C}^{C} \otimes C\right) \circ\left(\delta_{C} \otimes \delta_{C}\right) \circ\left(\sigma \otimes \mu_{C} \otimes \sigma^{-1} \otimes C\right) \circ \\
& \circ\left(C \otimes \tau_{C}^{C} \otimes C \otimes \lambda \otimes C \otimes \lambda\right) \circ\left(\delta_{C} \otimes \delta_{C} \otimes \delta_{C} \otimes C\right) \circ \\
& \circ\left(C \otimes C \otimes\left[\delta_{C} \circ \mu_{C}\right]\right) \circ\left(C \otimes \tau_{C}^{C} \otimes C\right) \circ\left(\delta_{C} \otimes \delta_{C}\right)= \\
& =\left(\sigma \otimes \mu_{C}\right) \circ\left(C \otimes \tau_{C}^{C} \otimes C\right) \circ\left(\delta_{C} \otimes \sigma^{-1} \otimes \delta_{C}\right) \circ \\
& \circ(C \otimes \lambda \otimes C \otimes \lambda) \circ\left(C \otimes \delta_{C} \otimes C\right) \circ\left(C \otimes \delta_{C}\right) \circ \delta_{C} \circ \\
& \circ\left(\sigma \otimes \mu_{C}\right) \circ\left(C \otimes \tau_{C}^{C} \otimes C\right) \circ\left(\delta_{C} \otimes \delta_{C}\right)= \\
& =\left(\sigma \otimes \mu_{C}\right) \circ\left(C \otimes \tau_{C}^{C} \otimes C\right) \circ\left(\delta_{C} \otimes \sigma^{-1} \otimes C \otimes C\right) \circ \\
& \circ\left(C \otimes \lambda \otimes C \otimes\left[(\lambda \otimes \lambda) \circ \tau_{C}^{C} \circ \delta_{C}\right]\right) \circ\left(C \otimes \delta_{C} \otimes C\right) \circ \\
& \circ\left(C \otimes\left[\tau_{C}^{C} \circ \delta_{C}\right]\right) \circ \delta_{C} \circ\left(\sigma \otimes \mu_{C}\right) \circ\left(C \otimes \tau_{C}^{C} \otimes C\right) \circ\left(\delta_{C} \otimes \delta_{C}\right)= \\
& =\left(\sigma \otimes C \otimes \sigma \sigma^{-1}\right) \circ\left(C \otimes \tau_{C}^{C} \otimes \lambda \otimes C\right) \circ \\
& \circ\left(C \otimes\left[\mu_{C} \circ(C \otimes \lambda) \circ \delta_{C}\right] \otimes \lambda \otimes \delta_{C}\right) \circ\left(C \otimes C \otimes \delta_{C}\right) \circ\left(C \otimes \delta_{C}\right) \circ \\
& \circ \delta_{C} \circ\left(\sigma \otimes \mu_{C}\right) \circ\left(C \otimes \tau_{C}^{C} \otimes C\right) \circ\left(\delta_{C} \otimes \delta_{C}\right)= \\
& =\left(\sigma \otimes \sigma^{-1} \otimes \eta_{C}\right) \circ(C \otimes \lambda \otimes \lambda \otimes C) \circ\left(\delta_{C} \otimes \delta_{C}\right) \circ \delta_{C} \circ \\
& \circ\left(\sigma \otimes \mu_{C}\right) \circ\left(C \otimes \tau_{C}^{C} \otimes C\right) \circ\left(\delta_{C} \otimes \delta_{C}\right)= \\
& =\left(\left[\partial_{1}\left(\sigma^{-1}\right) * \partial_{4}(\sigma)\right] \otimes \eta_{C}\right) \circ(C \otimes \lambda \otimes C) \circ\left(C \otimes \delta_{C}\right) \circ \delta_{C}^{\circ} \\
& \circ\left(\sigma \otimes \mu_{C}\right) \circ\left(C \otimes \tau_{C}^{C} \otimes C\right) \circ\left(\delta_{C} \otimes \delta_{C}\right)= \\
& =\left(\left[\partial_{2}\left(\sigma^{-1}\right) * \partial_{3}(\sigma)\right] \otimes \eta_{C}\right) \circ(C \otimes \lambda \otimes C) \circ\left(C \otimes \delta_{C}\right) \circ \delta_{C} \circ \\
& \circ\left(\sigma \otimes \mu_{C}\right) \circ\left(C \otimes \tau_{C}^{C} \otimes C\right) \circ\left(\delta_{C} \otimes \delta_{C}\right)= \\
& =\left(\sigma^{-1} \otimes \sigma \otimes \eta \eta_{C}\right) \circ\left(\mu_{C} \otimes \tau_{C}^{C} \otimes \mu_{C}\right) \circ\left(C \otimes \tau_{C}^{C} \otimes \tau_{C}^{C} \otimes C\right) \circ \\
&
\end{aligned}
$$




$$
\begin{aligned}
& \circ\left(\delta_{C} \otimes\left[(\lambda \otimes \lambda) \circ \tau_{C}^{C} \circ \delta_{C}\right] \otimes \delta_{C}\right) \circ\left(C \otimes \delta_{C}\right) \circ \delta_{C} \circ \\
& \circ\left(\sigma \otimes \mu_{C}\right) \circ\left(C \otimes \tau_{C}^{C} \otimes C\right) \circ\left(\delta_{C} \otimes \delta_{C}\right)= \\
& =\left(\sigma \otimes \sigma^{-1} \otimes \eta_{C}\right) \circ\left(C \otimes \tau_{C}^{C} \otimes C\right) \circ \\
& \circ\left(C \otimes [ \mu _ { C } \circ ( C \otimes \lambda ) \circ \delta _ { C } ] \otimes \left[\mu_{C} \circ(\lambda \otimes C) \circ\right.\right. \\
& \left.\left.\circ \delta_{C}\right] \otimes C\right) \circ\left(\delta_{C} \otimes \delta_{C}\right) \circ \delta_{C} \circ \\
& \circ\left(\sigma \otimes \mu_{C}\right) \circ\left(C \otimes \tau_{C}^{C} \otimes C\right) \circ\left(\delta_{C} \otimes \delta_{C}\right)= \\
& =\eta_{C} \circ \sigma
\end{aligned}
$$

because $\sigma$ is a 2-cocyle and $\mathrm{H}$ a cocommutative Hopf algebra.

If $\left[\left(\mathbf{B}, \rho_{B}\right)\right],\left[\left(\mathbf{B}^{\prime}, \rho_{B^{\prime}}\right)\right] \in \mathbf{N}_{C}(\mathbf{H})$ then the morphism $(f \otimes g) \circ \delta_{C}:$ $C \rightarrow B \otimes B^{\prime}$ factors through the equalizer $i_{B B^{\prime}}$, where $f$ and $g$ are the morphisms defined in Proposition 9 for $\left(\mathbf{B}, \rho_{B}\right)$ and $\left(\mathbf{B}^{\prime}, \rho_{B^{\prime}}\right)$ respectively. Let $h: C \rightarrow B \circ B^{\prime}$ be this factorization. The morphism $h$ is in $\operatorname{Reg}\left(C, B \circ B^{\prime}\right)$ with inverse the factorization of the morphism $\left(f^{-1} \otimes g^{-1}\right) \circ \delta_{C}$ through the equalizer $i_{B B^{\prime}}$, and satisfying $h \circ \eta_{C}=\eta_{B B^{\prime}}$. Moreover, $\bar{\sigma}_{f} * \bar{\sigma}_{g}=\bar{\sigma}_{h}$.

Remark. If $\mathcal{C}=K-\operatorname{Mod}$ ( $K$ a field), this result has been obtained by Sweedler for the $\mathbf{H}$-module algebra $\left(K, \varepsilon_{H} \otimes K\right)$ in [21].

12. Definition. A monoid $\mathbf{A}=\left(A, \eta_{A}, \mu_{A}\right)$ is said to be Azumaya if and only if:

i) $A$ is a progenerator in $C$.

ii) The morphism of monoids $\chi_{A}: A \otimes A \rightarrow[A, A] ; \chi_{A}:=\left[A, \mu_{A} \circ\right.$ $\left.\left(A \otimes \mu_{A}\right) \circ\left(\tau_{A}^{A} \otimes A\right)\right] \circ \alpha_{A}(A \otimes A)$ is an isomorphism.

13. Definition. On the set of $\mathbf{H}$-module monoid isomorphism classes of $\mathbf{H}$-module Azumaya monoids we define the following equivalence relation:

$$
\left(\mathrm{A}, \varphi_{A}\right) \sim\left(\mathrm{B}, \varphi_{B}\right) \Longleftrightarrow \mathrm{AE}(M)^{\mathrm{op}} \cong \mathrm{BE}(N)^{\mathrm{op}}
$$

for some progenerators $\mathbf{H}$-modules $\left(M, \varphi_{M}\right)$ and $\left(N, \varphi_{N}\right)$.

The set of equivalence classes of $\mathbf{H}$-module Azumaya monoids forms a group under the operation induced by the tensor product, $\left(\mathrm{AB}, \varphi_{A \otimes B}=\right.$ $\left.\left(\varphi_{A} \otimes \varphi_{B}\right) \circ\left(C \otimes \tau_{A}^{C} \otimes B\right) \circ\left(\delta_{C} \otimes A \otimes B\right)\right)$. The unit element is the class of the $\mathbf{H}$-module Azumaya monoid:

$$
\begin{aligned}
& \left(E(M)^{\circ p}, \varphi_{E(M)}=\left[M, \varphi_{M} \circ\left(C \otimes \beta_{M}(M)\right) \circ\left(\tau_{C}^{M} \otimes[M, M]\right) \circ\right.\right. \\
& \circ\left(\varphi_{M} \otimes C \otimes[M, M]\right) \circ\left(\tau_{C}^{M} \otimes C \otimes[M, M]\right) \circ\left(M \otimes \tau_{C}^{C} \otimes[M, M]\right) \circ \\
& \left.\left.\circ(M \otimes C \otimes \lambda \otimes[M, M]) \circ\left(M \otimes \delta_{C} \otimes[M, M]\right)\right] \circ \alpha_{M}(C \otimes[M, M])\right)
\end{aligned}
$$

for some progenerator $\mathbf{H}$-module $\left(M, \varphi_{M}\right)$; and the opposite of $\left(A, \varphi_{A}\right)$ is $\left(A^{\circ \mathrm{p}}, \varphi_{A}\right)$. This group is denoted by $\mathrm{BM}(\mathcal{C}, \mathrm{H})$. 
If $1=\left(1,1, \tau^{K}, 1\right)$ is the trivial Hopf algebra in $\mathcal{C}$, then we define the Brauer group of Azumaya monoids in $\mathcal{C}$ as $\mathrm{BM}(\mathcal{C}, 1)$ and we will denote it by $\mathrm{B}(\mathcal{C})$.

\section{Examples.}

1) If $\mathcal{C}$ is the category of modules over a commutative ring $R$, then $B(C)$ is the Brauer group of $R$ defined by Auslander and Goldman in $[4]$.

2) If $\mathcal{C}$ is the category of sheaves of $\theta$-modules, $B(C)$ is the Brauer group defined by Auslander in [3].

3) If $\mathcal{C}$ is the category of $(R, \sigma)$-Mod with $\sigma$ an idempotent noetherian kernel functor in $R$-Mod, López and Villanueva obtain, in [17], a homomorphism $B r(R, \sigma) \rightarrow B((R, \sigma)$-Mod $)$ which is an isomorphism if $R$ is a noctherian ring, where $\operatorname{Br}(R, \sigma)$ is the relative Brauer group introduced by Oystaeyen and Verschoren in $[22]$.

14. Definition. We denote by $\mathrm{BM}_{\mathrm{inn}}(\mathcal{C}, \mathrm{H})$ the subgroup of $\mathrm{BM}(\mathcal{C}, \mathbf{H})$ built, up with the equivalence classes that can be represented by an $\mathrm{H}$-module Azumaya monoid with inner action.

$([2,16])$.

15. Theorem. $\mathrm{BM}_{\mathrm{inn}}(\mathcal{C}, \mathrm{H}) \cong \mathrm{B}(\mathcal{C}) \oplus H^{2}(\mathrm{H}, K)$.

Proof:

The sequence

$$
1 \longrightarrow \mathrm{B}(\mathcal{C}) \stackrel{i}{\longrightarrow} \mathrm{BM}_{\mathrm{inn}}(\mathcal{C}, \mathbf{H}) \stackrel{\Pi}{\longrightarrow} \mathrm{N}_{C}(\mathbf{H}) \longrightarrow 1
$$

is split exact, where the morphism $i$ is given by $i[(A)]=\left[\left(A, \varepsilon_{C} \otimes A\right)\right]$ and the morphism $\Pi$ is given by $\Pi\left[\left(A, \varphi_{A}\right)\right]:=\left(\Pi(\mathbf{A}), \rho_{\Pi(A)}\right)$ with $\Pi(\mathbf{A}):=$ $\operatorname{Ig}(m, n)$

$$
\Pi(A) \stackrel{j}{\longrightarrow} A \otimes C \underset{n}{\stackrel{m}{\rightrightarrows}}[A, A \otimes C]
$$

where

$$
\begin{aligned}
m & =\left[A, \mu_{A} \otimes C\right] \circ \alpha_{A}(A \otimes C) \\
n & =\left[A,\left(\mu_{A} \otimes C\right) \circ\left(A \otimes\left(\varphi_{A} \circ \tau_{C}^{A}\right) \otimes C\right) \circ\left(\tau_{A}^{A} \otimes \delta_{C}\right)\right] \circ \alpha_{A}(A \otimes C)
\end{aligned}
$$

$([2,17])$.

If $H$ is a finitely generated projective, cormmutative and cocommutative Hopf algebra over a commutative ring $K$, this result generalizes the one obtained by Beattie in [5], and if the action of $H$ over $A$ is inner, the description of $\Pi(A)$ is due to Beattie and Ulbricht $([6])$. 


\section{References}

1. Alonso Álvarez, I. N., Extensiones Cleft en categorías cerradas. Interpretación cohomológica, Alxebra 58 (1992), Depto. Alxebra, Santiago de Compostela.

2. Alonso Álivarez, J. N. AND Ferníndez VilaboA, J. M., Inner actions and Galois $\mathbf{H}$-objects in a symmetric closed category, Depto. Alxebra, Santiago de Compostela (Preprint).

3. Auslander, M., The Brauer group of a ringed space, Journal of Algebra 4 (1966), 220-273.

4. Auslander, M. and Goldman, O., The Brauer group of a commutative ring, Transactions A.M.S. 97 (1960), 367-409.

5. BEATTIE, M., A direct sum decomposition for the Brauer group of $H$-module algebras, Journal of Algebra 43 (1976), 686-693.

6. Beattie, M. and Ulbricht, K. H., A Skolem-Noether theorem for Hopf algebra actions, Comm. in Algebra 18 (1990), 3713-3724.

7. Blattner, R. J. and Montgomery, S., Crossed products and Galois extensions of Hopf algebras, Pacific J. of Math. 137 (1989), $37-54$.

8. Caenepeel, S., A note on inner actions of Hopf algebras, Proc. A.M.S. 113, no. 1 (1991).

9. Chase, S. U. And Sweedler, M. E., "Hopf algebras and Galois theory:" Lect. Notes in Math. 97, 1969.

10. DoI, Y., Equivalent crossed products for a Hopf algebra, Communications in Algebra 17 (1989), 3053-3085.

11. EARLY, T. E. A.ND KREIMER, H. F., Galois algebras and Harrison cohomology, Journal of Algebra 58 (1979), 136-147.

12. Eilenberg, S. and Kelly, G. M., Closed categories, Proc. of the Conference on Categorical Algebra, La Jolla (1966), 421-562.

13. Fernández-Vilaboa, J. M., Grupos de Brauer y de Galois de un álgebra de Hopf en una categoría cerrada, Alxebra 42 (1985), Depto. Alxebra, Santiago de Compostcla.

14. Fernández-Vilabon, J. M. and López-López, M. P., Cleft comodule triples in a symmetric closed category, Bull. Soc. Math. Belgique, ser. B 40 (1988), 283-297.

15. IEREMías-López, A., El grupo fundamental relativo. Teoría de Galois y localización, Alxebra 56 (1991), Depto. Alxebra, Santiago de Compostela.

16. KReimer, H. F. AND TAKEuCh, M., Hopf algebras and Galois extensions of an algebra, Indiana Univ. Math. J. 30 (1981), 675-692. 
17. López-López, M. P. And Villanueva-NovoA, E., The Brauer group of the category $(R, \sigma)$-Mod. (An alternative to the theory of relative invariants), Proc. of the first Belgian-Spanish week on AIgebra and Geometry (1988), Rijksuniversitair Centrum Antwerpen Groenenborgelaan 171, B-2020 Antwerpen, Belgium, 75-93.

18. MAC LANE, S., "Categories for the working mathematicien," G.T.M. 5, Springer, 1971.

19. Pachuashvili, B., Cohomologies and extensions in monoidal categories, Journal of Pure and Applied Algebra 72 (1991), 109-147.

20. PAREIGIS, B., "Non-additive ring and module theory IV: The Brauer group of a symmetric monoidal category," Lecture Notes in Math. 549, 1976, pp. 122-133.

21. SWEEdler, M. E., Cohomology of algebras over Hopf algebras, Transactions of the A.M.S. 133 (1968), 205-239.

22. Van OystaEyen, F. AND Verschoren, A., "Relative invariants of rings. The commutative theory:" Lect. Notes in Pure and App. Math. 79, Marcel Dekker, New York, 1983.

23. Yokogawa, K., The cohomological aspects of Hopf-Galois extensions over a commutative ring, Osaka J. Moth. 18 (1981), 75-93.

J. N. Alonso Álvarez:

Departamento de Matemáticas

Universidad de Vigo

Lagoas-Marcosende

E-36271 Vigo

SPAIN
J. M. Fernández Vilaboa:

Departamento de Alxebra

Universidad de Santiago de Compostela

E-15771 Santiago de Compostela

SPAIN

Primera versió rebuda el 17 d'Agost de 1992, darrera versió rebuda el 27 d'Abril de 1993 